\title{
Caloric restriction increases internal iliac artery and penil nitric oxide synthase expression in rat: Comparison of aged and adult rats
}

\author{
Emin Ozbek $^{1}$, Abdulmuttalip Simsek ${ }^{1}$, Mustafa Ozbek ${ }^{2}$, Adnan Somay ${ }^{3}$ \\ ${ }^{1}$ Okmeydani Research and Education Hospital, Department of Urology, Istanbul, Turkey; \\ ${ }^{2}$ Diskapi Research and Education Hospital, Department of Endocrinology, Ankara, Turkey; \\ ${ }^{3}$ Fatih Sultan Mehmet Research and Education Hospital, Department of Pathology, Istanbul, Turkey.
}

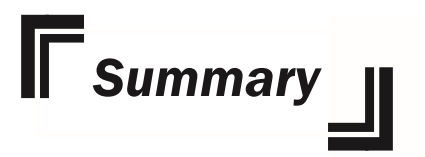

Because of the positive corelation between healthy cardiovascular system and sexual life we aimed to evaluate the effect of caloric restriction (CR) on endothelial and neuronal nitric oxide synthase (eNOS, nNOS) expression in cavernousal tissues and eNOS expression in the internal iliac artery in young and aged rats. Young $(3 \mathrm{mo}, n=7)$ and aged (24 mo, $n=7$ ) male Sprague-Dawley rats were subjected to $40 \% \mathrm{CR}$ and were allowed free access to water for 3 months. Control rats $(n=14)$ fed ad libitum had free access to food and water at all times. On day 90, rats were sacrified and internal iliac arteries and penis were removed and parafinized, eNOS and nNOS expression evaluated with immunohistochemistry. Results were evaluated semiquantitatively. eNOS and nNOS expression in cavernousal tissue in CR rats were more strong than in control group in both young and old rats. eNOS expression was also higher in the internal iliac arteries of CR rats than in control in young and old rats. As a result of our study we can say that there is a positive link between $C R$ and neurotransmitter of erection in cavernousal tissues and internal iliac arteries. CR has beneficial effect to prevent sexual dysfunction in young and old animals and possible humans.

KEY WORDS: Rat; Caloric restriction; Nitric oxide synthase; Internal iliac artery; Penis.

\begin{abstract}
INTRODUCTION
The incidence of erectile dysfunction (ED) increases with age. It is reported that $35 \%$ of married men aged 60 years and older suffer from ED (1). From the prevalence rates reported in the Massachusetts Male Aging Study (MMAS), between the ages of 40 and 70 years, the probability of complete ED increased from $5.1 \%$ to $15 \%$, moderate dysfunction increased from $17 \%$ to $34 \%$, and mild ED remained constant at about 17\% (1). According to the study of the National Health and Social Life Survey (NHSLS) the following prevalence rates for ED were reported (responses to questions regarding obtaining and maintaining erection): $7 \%$ for ages 18 to 29 years, $9 \%$ for ages 30 to $39,11 \%$ for ages 40 to 49 , and $18 \%$ for 50 to 59 (1). Obesity has become a worldwide public health problem, it may decrease life expectancy by 7 years at the age of
\end{abstract}

40 years: excess bodyweight is now the sixth most important risk factor contributing to the overall burden of disease throught the world. Overweight and obesity may increase the risk of ED by 30-90\% as compared with normal subjects. Moreover, women with the metabolic syndrome have an increased prevalence of sexual dysfunctions as compared with matched controls. Lifestyle changes reducing body weight induces amelioration of both erectile and endothelial functions in obese men (2). Patients with ED show a higher body mass index (BMI), waist circumference (WC), and insulin-resistance (IR) and lower levels of total testosterone (TT ) and bioavailable testosterone (BT). There is a negative correlation between erectile function and IR and abdominal obesity. The TT levels are lower in patients with increased BMI, 
WC and IR. Negative correlation was shown only between BT and abdominal obesity (3). Androgen deficiency together with endothelial dysfunction may be responsible from ED in obesity (4).

In animal experiments penile endonhelial nitric oxide synthase (eNOS) and neuronal nitric oxide synthase (nNOS) expression were found decreased in hypercholesterolemic cavernousal tissue due to decrease activity of AMP-activated protein kinase (AMPK), which increases the expression of neuronal (n) NOS and endothelial (e) NOS (5).

In another experiment we shown that mild to moderate exercise increases penile eNOS and nNOS expression as well as serum total testosterone levels in young and aged rats (6).

Modifiable lifestyle factors such as obesity, lack of exercise and smoking play a role in the development, progression or remission not only of erectile dysfunction (ED), but also in cardiovascular disease and the metabolic syndrome. One-third of obese men with ED can regain their sexual activity after 2 y of adopting health behaviors, mainly regular exercise and reducing weight. Western societies actually spend a huge part of their health care costs on chronic disease treatment and interventions for risk factors. The adoption of healthy lifestyles can reduce the prevalence of obesity and the metabolic syndrome, and hopefully the burden of sexual dysfunction (7).

Mediterranean-style diets and a reduction in caloric intake have been found to improve erectile function in men with the aspects of the metabolic syndrome. In addition, both clinical and experimental studies have confirmed that combining the two interventions provides additional benefit to erectile function, likely via reduced metabolic disturbances (e.g., inflammatory markers, insulin resistance), decreased visceral adipose tissue, and improvement in vascular function (e.g., increased endothelial function) (8).

Mediterranean-style diet might be effective in ameliorating sexual function in women with metabolic syndrome. Lifestyle changes, mainly focussing on regular physical activity and a healthy diet, are effective and safe ways to reduce cardiovascular diseases and premature mortality in all population groups; they may also prevent and treat sexual dysfunctions in both sexes (9).

Taking all of these background into account, the objective of the our work was to investigate the role of caloric restriction on eNOS as well as nNOS and eNOS expression in the internal iliac artery and cavernousal tissue of young and aged rats, respectively.

\section{Materials AND Methods}

\section{Animals and diet}

3-month-old young and 24-month-old aged male Spraque-Dawley rats were divided into four experimental groups ( $\mathrm{n}=7$ rats per group). Control rats in each group were fed ad libitum with pelleted standard diet. Another two groups were subjected to $40 \%$ caloric restriction for three months (10).

Rats were housed induvidually with free access to water in wire- bottom cages and acclimated at $22^{\circ} \mathrm{C}$ with a 12 $\mathrm{h}$ light/dark cycle. Caloric restricted rats were fed on a daily basis at the beginning of the dark cycle and the amount of food was weekly updated. All animal experiments were approved by the Animal Ethics Committee.

Isolation of samples and immunohistochemical staining At the end of 3 months rats were sacrificed and the and internal iliac arteries and penises were quickly removed, washed with saline and parafinized. All procedures were performed under general anaesthesia with $50 \mathrm{mg} / \mathrm{kg}$ ketamine HCL administered intraperitoneally. eNOS, nNOS expression in all tissues was evaluated with immunohistochemistry using specific antibodies.

For the immunohistochemical evaluation, specimens were processed for light microscopy and sections incubated at $+4^{\circ} \mathrm{C}$ overnight and then de-waxed in xylene for $30 \mathrm{~min}$. After rehydrating in a decreasing series of ethanol, sections were washed with distilled water and phosphate buffered saline (PBS) for $10 \mathrm{~min}$. Sections were then treated with $2 \%$ trypsin in $50 \mathrm{~mm}$ Tris buffer $(\mathrm{pH} 7.5)$ at $37^{\circ} \mathrm{C}$ for $15 \mathrm{~min}$ and washed with PBS. Sections were delineated with a Dako pen (Dako, Glostrup, Denmark) and incubated in a solution of 3\% $\mathrm{H} 2 \mathrm{O} 2$ for $15 \mathrm{~min}$ to inhibit endogenous peroxidase activity. Then, sections were incubated with $e$ NOS Ab-1 (RB-9279-R7, Neomarkers, Labvision, Fremont, CA, USA) and nNOS (sc-648, Santa Cruz Biotechnology Inc., Santa Cruz, CA, USA) antibodies.

The Ultra-vision (Labvision) horseradish peroxidase/3amino-9-ethylcarbazole staining protocol was used at this stage.

Sections prepared for each case were examined by light microscopy. Positive and negative controls were conducted in parallel with NOS stained sections. Staining of sections with commercially available antibodies served as the positive control.

Negative controls included staining tissue sections with omission of the primary antibody.

The sections were evaluated for diffuseness and staining. Penile eNOS and nNOS were evaluated according to the diffuseness and intensity of staining in penile cavernousal smooth muscle.

According to the diffuseness of the staining, sections were graded as $0=$ no staining; $1=$ staining $<25 \% ; 2=$ staining $25-50 \% ; 3=$ staining $50-75 \% ; 4=$ staining $>$ $75 \%$. According to staining intensity, sections were graded as follows: 0 = no staining; 1 = weak but detectable staining; 2 = distinct; 3 = intense staining $(11,6)$. Immunohistochemical values were obtained by adding the diffuseness and intensity scores.

\section{RESULTS}

eNOS expression in internal iliac artery: In control young and aged rat internal iliac arteries there was focal mild to moderate e NOS expression, but diffuse in caloric restricted young and aged rats (Figure 1A-D). eNOS and nNOS expression in cavernousal tissue: eNOS, nNOS expression were weak in the cavernousal tissues of control rats. In caloric restricted group eNOS, nNOS expression were more evident than in control young and aged rats (Figure 2A-H). 
Figure 1A-C.

eNOS expression in internal iliac artery.

A-B: Young

Control Group:

Focal mild internal

iliac artery

eNOS staining

(IHC 400X).
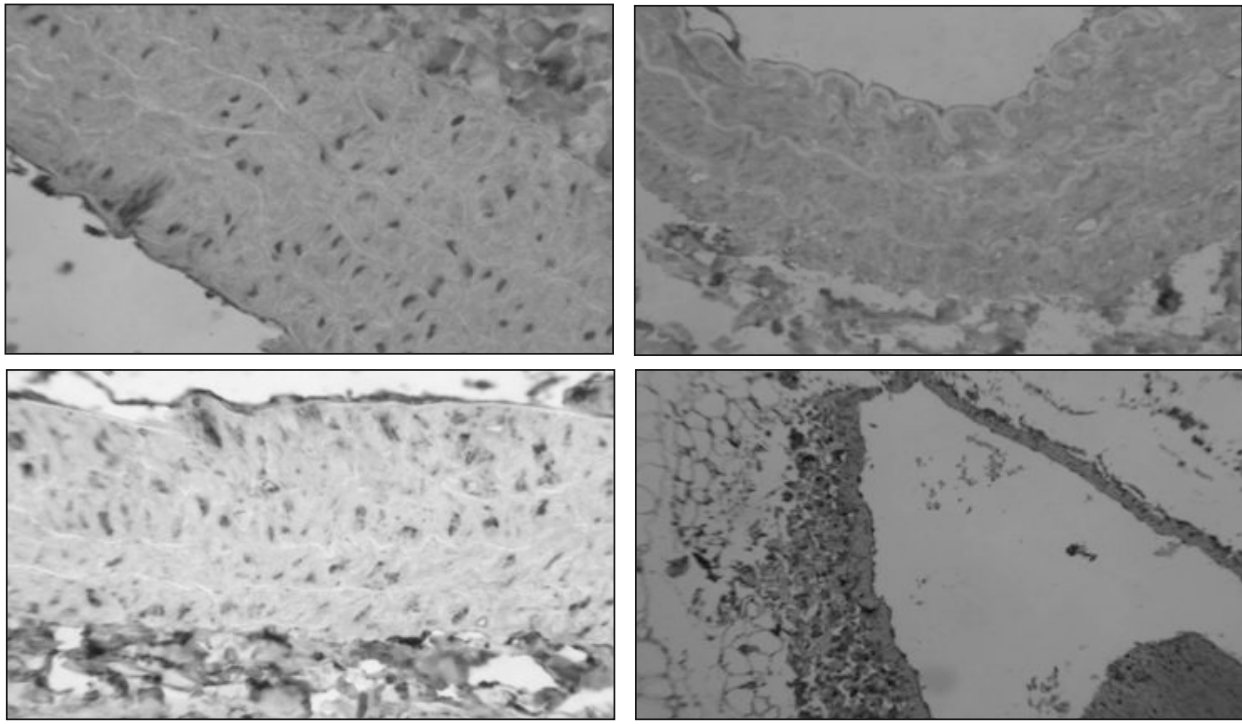

C-D: Young Caloric

Restriction Group:

Diffuse internal

iliac artery

eNOS staining

(IHC 400X).

Figure 2A-H.

eNOS and nNOS expression in cavernousal tissue.

A: Young

Control Group:

Focal mild penile

eNOS staining

(IHC 400X).

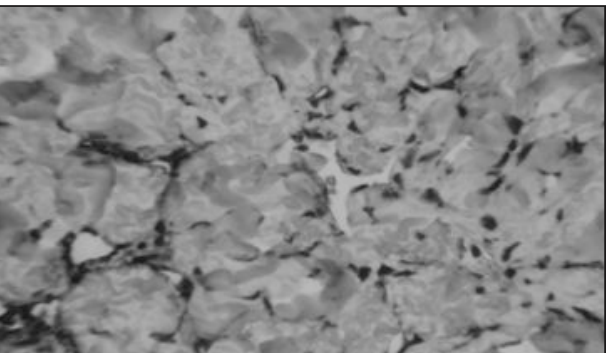

C: Young

Caloric Restriction

Group: Diffuse

penile eNOS

staining

(IHC 400X).

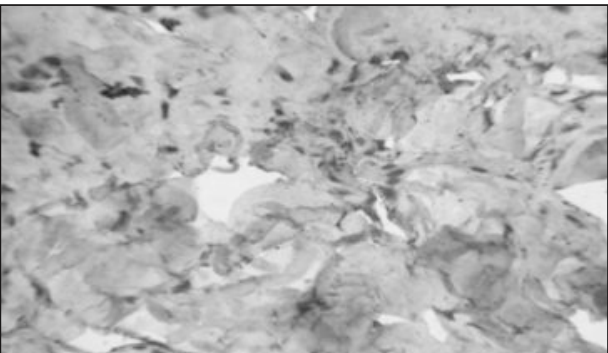

E: Young

Control Group:

Focal mild penile

$n N O S$ staining

(IHC 400X).

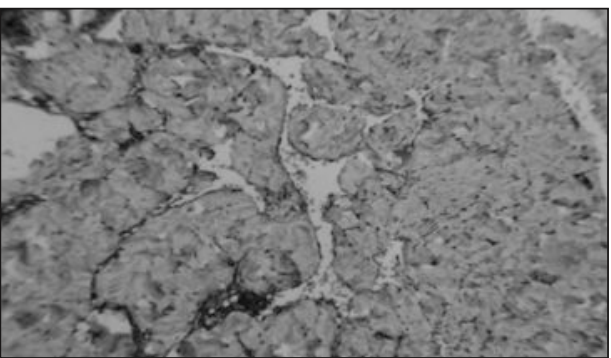

G: Young

Caloric Restriction

Group: Diffuse

penile nNOS

staining

(IHC 400X).

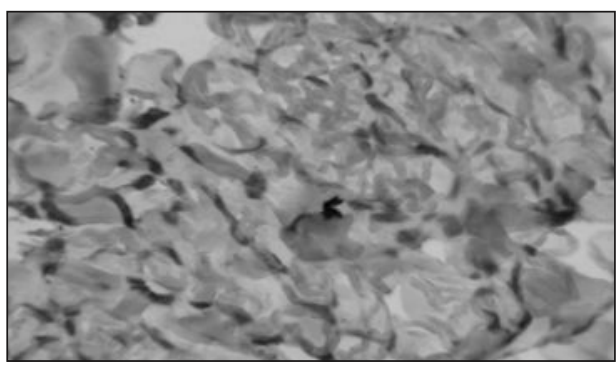

B: Aged

Control Group:

Focal mild penile eNOS staining

(IHC 400X).

D: Aged

Caloric Restriction

Group: Diffuse

penile eNOS

staining

(IHC 400X).

F: Aged

Control Group:

Focal mild penile

nNOS staining

(IHC 400X).

H: Aged

Caloric Restriction

Group: Diffuse

penile nNOS

staining

(IHC 400X). 


\section{Discussion}

Erectile function is a multi system phenomenon involving vascular, neuronal and endocrin system. In this process nitric oxide (NO) released from nerve endings and endothelial cells plays a key role. NO is produced from L-arginine through an enzymatic reaction in which the enzyme nitric oxide synthase is involved. In the cavernousal tissue NO stimulates guanilate cyclase enzyme present in the smooth muscle cells. Guanylate cyclase induces the formation of cyclicguanosine monophosphate (GMPC) from guanosine triphosphate (GTP). Phosphorilation of GMPC, results in cytoplasmic calcium release causing smooth muscle relaxation of the corpus cavernousum, with the subsequent penile tumescence $(12,13)$.

The most common causes of ED are organic such as cardiovascular and endocrin diseases including obesity, type-2 diabetes mellitus (DM2) and metabolic syndrome.Depression, hormonal changes and vascular or neurological damage after trauma or surgery are other factors aasociated with $\operatorname{ED}(3,14)$.

Obesity causes insulin resistence and cardiovascular system diseases through disrupting in the signaling pathways required for nitric oxide production with subsequently endothelial dysfunction. Nowadays obesity is a major health problem throught the world, especially in Western countries. Type 2 diabetes mellitus, hypertension, hyperlipidemia are comorbitidies associated with obesity that cause cardiovascular disease and endothelial dysfunction. These abnormalities are frequently clustered in the so called "metabolic syndrome". Obesity and metabolic syndrome may lead directly to endothelial dysfunction and subsequently erectile dysfunction (15). Villalba et al. reported that endothelial relaxant responses were impaired in penile arteries of obese Zucker rats (16). Enhanced superoxide production and reduced basal NO activity are the proposed underlying mechanism in this process. In human, obesity causes impaired indices of endothelial function and increases circulating concentrations of the proinflammatory cytokines interleukin-6 (IL-6), interleukin-8 (IL-8), interleukin-18 (IL18), as well as C-reactive protein (CRP) and NO bioavailability (17). NOS expression in high- fat-fed obese rats has been found lower and restored by metformin (18). In our experiment we found e NOS and n NOS expression lower in hypercholesterolemic young and aged rats. Caloric restiction restriction restores NOS expression in both group. Reduced caloric intake decreases arterial pressure in healthy induviduals and improeves endothelium vasodilatation in obese and overweight induviduals. In literature it is reported that caloric restriction promotes endothelium- dependent vascular relaxation by activating e NOS activity in mice throught SIRT1 (19). In our experiment we found that caloric restiction increases e NOS expression in the internal iliac artery of rats. Because penile arterial supply comes from internal iliac artery we can say that caloric restriction improves penile blood supply by increasing internal artery vasodilatation. In vitro experiments are required to demonstrate the effect of caloric restriction on the endothelial relaxant response of caloric restiction in the internal iliac artery. Weight loss resulting from CR improves endothelium- dependent vascular relaxation in obese and overweight induviduals with hypertension $(20,21)$. In this experiment, Authors show that SIRT1 promotes endotheliumdependent vasodilation by targeting endothelial nitric oxide synthetase (eNOS) for deacetylation. SIRT1 and eNOS co-localize and co-precipitate in endothelial cells, and SIRT1 deacetylates eNOS, stimulating eNOS activity and increasing endothelial nitric oxide (NO). These mechanisms may be effective in the internal ilac arteries and cavernousal endothelial cells. Further studies are needed to confirm this suggestion.

Caloric restriction improves cardiovascular system through increase of systemic NO release, increase of NO bioavailability, upregulation of sirtuin-1 as well as reducing oxidative stress in animal models. Recently, it is reported that 8 weeks 30\% caloric restriction reverses vascular endothelial dysfunction in old mice by restoring NO bioavailability, reducing oxidative stress (via reduced NADPH oxidase-mediated superoxide production and stimulation of anti-oxidant enzyme activity) and upregulation of sirtuin-1 (22). In another study it was found that, $\mathrm{CR}$ reduce blood pressure by elevating $\mathrm{NO}$ production and lowering ACE activity in rats (23). Shinmura et al. reported that prolonged (6 months) CR improves myocardial ischemic tolerance and restores the ischemic precontidioning effect in middle-aged rats through nitric oxide-dependent increase in nuclear SIRTl content (24). Nisoli et al. report that caloric restriction for either 3 or 12 months induced endothelial nitric oxide synthase (eNOS) expression and 3',5'-cyclic guanosine monophosphate formation in various tissues of male mice. Other Authors stated that this was accompanied by mitochondrial biogenesis, with increased oxygen consumption, adenosine triphosphate production and enhanced expression of sirtuin 1 (25). In different experiments it was shown that $\mathrm{CR}$ increases aortic eNOS and NO release as well as improves endotheliumdependent vasorelaxation to acetylcholine (26).

In a clinical study caloric restriction improves endothelial-dependent vasodilation through an increased release of nitric oxide in obese hypertensive patients (27).

As a conclusion, as it shown in literature, CR improves cardiovascular system by increasing NO levels, NO bioavailability as well as decreasing ROS, proinflammatory and inflammatory cytokines. Our study is the first to demonstrate the local effect of caloric restriction in the pathophysiology of ED at molecular level. Further in vitro studies are needed to evaluate the contractionrelaxation responses of cavernousal and internal iliac artery strips in CR rats. In clinical practice we think that CR improves response to phosphodiesterase- 5 inhibitors in aged and young subjects. Further clinical studies are also needed to confirm this suggestion.

\section{References}

1. Wein AJ, Kavoussi LR, Novick AC, et al. Campbell-Walsh Urology, Ninth edition, Elsevier, Philadelphia, vol. 1, p.738.

2. Esposito K, Giugliano F, Ciotola M, et al. Obesity and sexual dysfunction, male and female.Int J Impot Res. 2008; 20:358-65. 
3. Knoblovits P, Costanzo PR, Rey Valzacchi GJ, et al. Erectile dysfunction, obesity, insulin resistance, and their relationship with testosterone levels in eugonadal patients in an andrology clinic setting. J Androl. 2010; 31:263-70.

4. Traish AM, Feeley RJ, Guay A. Mechanisms of obesity and related pathologies: androgen deficiency and endothelial dysfunction may be the link between obesity and erectile dysfunction. FEBS J. 2009; 276:5755-67.

5. Kim YW, Park SY, Kim JY, et al. Metformin restores the penile expression of nitric oxide synthase in high-fat-fed obese rats. J Androl. 2007; 28:555-60.

6. Ozbek E, Tasci AI, Ilbey YO, et al. The effect of regular exercise on penile nitric oxide synthase expression in rats. Int J Androl. 2010, 33:623-8.

7. Esposito K, Giugliano D. Obesity, the metabolic syndrome, and sexual dysfunction. Int J Impot Res. 2005; 17:391-8.

8. Hannan JL, Maio MT, Komolova M, Adams MA. Beneficial impact of exercise and obesity interventions on erectile function and its risk factors. J Sex Med. 2009; 6(Suppl 3):254-61.

9. Esposito K, Giugliano F, Ciotola M, et al. Obesity and sexual dysfunction, male and female. Int J Impot Res. 2008; 20:358-65.

10. Zanetti M, Barazzoni R, Vadori M, et al. Lack of direct effect of moderate hyperleptinemia to improve endothelial function in lean rat aorta: role of calorie restriction. Atherosclerosis. 2004; 175:253-9.

11. Moochhala S, Chhatwal VJ, Chan ST, et al. Nitric oxide synthase activity and expression in human colorectal cancer. Carcinogenesis 1996; 17:1171-1174.

12. Barouch LA, Harrison RW, Skaf MW, et al. Nitric oxide regulates the heart by spatial confinement of nitric oxide synthetase isoforms. Nature. 2002; 214:337-339.

13. Trussell JC, Legro RS. Erectile dysfunction: does insulin resistance play a part? Fertil Steril. 2007; 88:771-777.

14. Costanzo P, Knoblovits P, Rey Valzacchi G, et al. Erectile dysfunction is associated with a high prevalence of obesity and metabolic syndrome. Rev Argent Endocrinol Metab. 2008; 45:142-148.

15. Fonseca V, Jawa A. Endothelial and erectile dysfunction, diabetes mellitus, and the metabolic syndrome: common pathways and treatments? Am J Cardiol. 2005; 96(12B):13M-18M.
16. Villalba N, Martínez P, Bríones AM, et al. Differential structural and functional changes in penile and coronary arteries from obese Zucker rats. Am J Physiol Heart Circ Physiol. 2009; 297:H696-707.

17. Giugliano F, Esposito K, Di Palo C, et al. Erectile dysfunction associates with endothelial dysfunction and raised proinflammatory cytokine levels in obese men. J Endocrinol Invest. 2004; 27:665-9.

18. Kim YW, Park SY, Kim JY, et al. Metformin restores the penile expression of nitric oxide synthase in high-fat-fed obese rats. J Androl. 2007; 28:555-60.

19. Mattagajasingh I, Kim CS, Naqvi A, et al. SIRT1 promotes endothelium-dependent vascular relaxation by activating endothelial nitric oxide synthase. Proc Natl Acad Sci USA. 2007; 104:14855-60.

20. Raitakari M, Ilvonen T, Ahotupa M, et al. Weight reduction with very-low-caloric diet and endothelial function in overweight adults: role of plasma glucose. Arterioscler Thromb Vasc Biol. 2004; 24:124-128.

21. Sasaki S, Higashi Y, Nakagawa K, et al. A low-calorie diet improves endothelium-dependent vasodilation in obese patients with essential hypertension. Am J Hypertens. 2002; 15:302-309.

22. Rippe C, Lesniewski L, Connell M, et al. Short-term calorie restriction reverses vascular endothelial dysfunction in old mice by increasing nitric oxide and reducing oxidative stress. Aging Cell. 2010; 9:304-12.

23. Sharifi AM, Mohseni S, Nekoparvar S, et al. Effect of caloric restriction on nitric oxide production, ACE activity, and blood pressure regulation in rats. Acta Physiol Hung. 2008; 95:55-63.

24. Shinmura K, Tamaki K, Bolli R. Impact of 6-mo caloric restriction on myocardial ischemic tolerance: possible involvement of nitric oxide-dependent increase in nuclear Sirt1. Am J Physiol Heart Circ Physiol. 2008; 295:H2348-55.

25. Nisoli E, Tonello C, Cardile A, et al. Calorie restriction promotes mitochondrial biogenesis by inducing the expression of eNOS. Science. 2005; 310:314-7.

26. Zanetti M, Barazzoni R, Vadori M, et al. Lack of direct effect of moderate hyperleptinemia to improve endothelial function in lean rat aorta: role of calorie restriction. Atherosclerosis. 2004; 175:253-9.

27. Sasaki S, Higashi Y, Nakagawa K, et al. A low-calorie diet improves endothelium-dependent vasodilation in obese patients with essential hypertension. Am J Hypertens. 2002; 15:302-9.

\author{
Correspondence \\ Emin Ozbek, MD (Corresponding Author) \\ ozbekemin@hotmail.com \\ Abdulmuttalip Simsek, MD \\ Department of Urology \\ Okmeydani Research and Education Hospital \\ Darulaceze street, 34384, Sisli, Istanbul, Turkey \\ Mustafa Ozbek, MD \\ Department of Endocrinology \\ Diskapi Research and Education Hospital \\ Ankara, Turkey \\ Adnan Somay, MD \\ Department of Pathology \\ Fatih Sultan Mehmet Research and Education Hospital \\ Istanbul, Turkey
}

